TRANSACTIONS OF THE

AMERICAN MATHEMATICAL SOCIETY

Volume 357, Number 5, Pages 2043-2058

S 0002-9947(04)03600-1

Article electronically published on November 4, 2004

\title{
ON THE THEORY OF ELLIPTIC FUNCTIONS BASED ON ${ }_{2} F_{1}\left(\frac{1}{3}, \frac{2}{3} ; \frac{1}{2} ; z\right)$
}

\author{
LI-CHIEN SHEN
}

\begin{abstract}
Based on properties of the hypergeometric series ${ }_{2} F_{1}\left(\frac{1}{3}, \frac{2}{3} ; \frac{1}{2} ; z\right)$, we develop a theory of elliptic functions that shares many striking similarities with the classical Jacobian elliptic functions.
\end{abstract}

The standard approach to the theory of the Jacobian elliptic functions begins with the development of the theory of the theta functions, and the elliptic functions are introduced in terms of the ratios of the theta functions. The crucial properties of the elliptic functions such as the differential equations associated with the elliptic functions and the connection with the hypergeometric series ${ }_{2} F_{1}\left(\frac{1}{2}, \frac{1}{2} ; 1 ; z\right)$ then follow from the general theory of the theta functions. In this work we consider the theory of elliptic functions from the perspective of the hypergeometric series ${ }_{2} F_{1}\left(\frac{1}{2}, \frac{1}{2} ; \frac{1}{2} ; z\right)$ and ${ }_{2} F_{1}\left(\frac{1}{3}, \frac{2}{3} ; \frac{1}{2} ; z\right)$.

The contents are organized as follows. In Section 1, we first list some of the crucial properties of the Jacobian elliptic functions, and then reformulate them in terms of ${ }_{2} F_{1}\left(\frac{1}{2}, \frac{1}{2} ; \frac{1}{2} ; z\right)$; this approach will provide not only the crucial motivation but also the road map for formulating a parallel theory of the elliptic function theory based on ${ }_{2} F_{1}\left(\frac{1}{3}, \frac{2}{3} ; \frac{1}{2} ; z\right)$. In Section 2 , we introduce three functions analogous to the ones encountered in the previous section and, based on the simple properties of ${ }_{2} F_{1}\left(\frac{1}{3}, \frac{2}{3} ; \frac{1}{2} ; z\right)$, we derive a corresponding set of formulas which share striking similarities with their counterparts. One of the functions will be represented in Section 3 in terms of the Weierstrass $\wp$ function, and additional properties are derived from the theory of conformal mappings. In Section 4, we list the theta functions and the essential formulas involving the theta functions and the Weierstrass $\wp$ function. The most technical aspect of the paper is in Section 5. It seems appropriate to insert a brief remark here. We recall that in the theory of the Jacobian elliptic functions, it is simple to establish that the theta quotient $\frac{\theta_{3}}{\theta_{2}} \frac{\theta_{1}\left(z / \theta_{3}^{2}\right)}{\theta_{4}\left(z / \theta_{3}^{2}\right)}$ satisfies the following differential equation [5, p. 491]:

$$
\left(y^{\prime}\right)^{2}=\left(1-y^{2}\right)\left(1-k^{2} y^{2}\right) \text { with } y(0)=1,
$$

where $k=\frac{\theta_{2}^{2}}{\theta_{3}^{2}}$. However, for a given $0<k<1$, it is very difficult to solve the above differential equation (the inverse problem). In fact, it is commented in 5, p. 492] that unless the theory of theta function is assumed, it is exceedingly difficult to even show that the above differential equation has an analytic solution except at simple poles. The main goal of this section is essentially to solve a similar

Received by the editors December 20, 2002 and, in revised form, December 15, 2003.

2000 Mathematics Subject Classification. Primary 11L05.

Key words and phrases. Jacobian elliptic functions, theta functions, Weierstrass $\wp$ function. 
inverse problem for a differential equation arising from the current theory with the help of the existing properties of the Weierstrass $\wp$ function. In Sections 6 and 7, we see that many interesting counterparts of the Jacobian elliptic functions follow naturally from the machinery established in the earlier sections.

The reader's familiarity with the subjects of the Weierstrass $\wp$ function, the basic properties of the theta functions and the classical Jacobian elliptic functions is assumed.

\section{BASIC PROPERTIES AND REFORMULATION OF THE JACOBIAN ELLIPTIC FUNCTIONS}

We first list some of the most important properties of the Jacobian elliptic functions. (See [5, Chapter XXII] for the definitions and the proofs of these identities.)

$$
\begin{aligned}
& s n^{2} u+c n^{2} u=1, \\
& k^{2} s n^{2} u+d n^{2} u=1 . \\
& s n^{\prime} u=c n u d n u, \\
& c n^{\prime} u=- \text { sn } u d n u, \\
& d n^{\prime} u=-k^{2} \text { snucnu. } \\
u= & \int_{0}^{s n u} \frac{d t}{\sqrt{\left(1-t^{2}\right)\left(1-k^{2} t^{2}\right)}} \\
= & \int_{d n u}^{1} \frac{d t}{\sqrt{\left(1-t^{2}\right)\left(t^{2}-k^{\prime 2}\right)}}
\end{aligned}
$$

(See [5. p. 492-493] for the proofs of (1.1)-(1.3).)

Also, $d n u$ satisfies the differential equation

$$
\left(d n^{\prime} u\right)^{2}=\left(1-d n^{2} u\right)\left(d n^{2} u-k^{\prime 2}\right) .
$$

Moreover, we have the Lambert series [5, p. 511, Example 1]

$$
\theta_{3}^{2} d n\left(\theta_{3}^{2} z\right)=1+4 \sum_{n=1}^{\infty} \frac{q^{n}}{1+q^{2 n}} \cos 2 n z
$$

For later comparison, we also mention [5, p. 493]

$$
\operatorname{sn} 0=0, \quad \text { cn } 0=1, \quad d n 0=1,
$$

and

$$
s n K=1, \quad c n K=0, \quad d n K=k^{\prime},
$$

where $k=\frac{\theta_{2}^{2}}{\theta_{3}^{2}}$ and $k^{\prime}=\frac{\theta_{4}^{2}}{\theta_{3}^{2}}$ are, respectively, the modulus and complementary modulus of the elliptic functions, and $K=\frac{\pi}{2} \theta_{3}^{2}$.

Thus, the identity

$$
\theta_{2}^{4}+\theta_{4}^{4}=\theta_{3}^{4}
$$

is precisely the identity

$$
k^{2} s n^{2} K+d n^{2} K=1
$$

We now reformulate the theory of the classical Jacobian elliptic functions from the perspective of the hypergeometric series ${ }_{2} F_{1}\left(\frac{1}{2}, \frac{1}{2} ; \frac{1}{2} ; x\right)$. This approach will form the basis for the development elliptic function theory based on ${ }_{2} F_{1}\left(\frac{1}{3}, \frac{2}{3} ; \frac{1}{2} ; x\right)$. 
We recall that the hypergeometric series ${ }_{2} F_{1}(a, b ; c ; z)$ is defined as

$$
{ }_{2} F_{1}(a, b ; c ; z)=\sum_{n=0}^{\infty} \frac{(a)_{n}(b)_{n}}{n !(c)_{n}} z^{n} .
$$

The following identity is well known [2] p. 101]:

$$
{ }_{2} F_{1}\left(\frac{1}{2}+\frac{a}{2}, \frac{1}{2}-\frac{a}{2} ; \frac{1}{2} ; \sin ^{2} z\right)=\frac{\cos a z}{\cos z} .
$$

Two special cases of the above identity corresponding to $a=0$ and $a=\frac{1}{3}$ are used in this work:

$$
\begin{aligned}
& { }_{2} F_{1}\left(\frac{1}{2}, \frac{1}{2} ; \frac{1}{2} ; \sin ^{2} z\right)=\frac{1}{\cos z}, \\
& { }_{2} F_{1}\left(\frac{1}{3}, \frac{2}{3} ; \frac{1}{2} ; \sin ^{2} z\right)=\frac{\cos \frac{z}{3}}{\cos z} .
\end{aligned}
$$

Define

$$
\begin{aligned}
u & =\int_{0}^{\varphi}{ }_{2} F_{1}\left(\frac{1}{2}, \frac{1}{2} ; \frac{1}{2} ; k^{2} \sin ^{2} \theta\right) d \theta \\
& =\int_{0}^{\sin \varphi}{ }_{2} F_{1}\left(\frac{1}{2}, \frac{1}{2} ; \frac{1}{2} ; k^{2} t^{2}\right) \frac{d t}{\sqrt{1-t^{2}}} \\
& =\int_{0}^{\sin \varphi} \frac{d t}{\sqrt{\left(1-t^{2}\right)\left(1-k^{2} t^{2}\right)}} \text { (from (1.7)). }
\end{aligned}
$$

Comparing with (1.3), we have

$$
\begin{gathered}
\sin \varphi=\operatorname{snu}, \\
\cos \varphi=\sqrt{1-\sin ^{2} \varphi}=\sqrt{1-s n^{2} u}=\operatorname{cnu},
\end{gathered}
$$

and

$$
\frac{d \varphi}{d u}=\frac{1}{{ }_{2} F_{1}\left(\frac{1}{2}, \frac{1}{2} ; \frac{1}{2} ; k^{2} s n^{2} u\right)}=\sqrt{1-k^{2} s n^{2} u}=d n u .
$$

Theorem 1.1. Let $0<k=\frac{\theta_{2}^{2}}{\theta_{3}^{2}}<1$. Define

$$
\theta_{3}^{2} z=\int_{0}^{\Phi}{ }_{2} F_{1}\left(\frac{1}{2}, \frac{1}{2} ; \frac{1}{2} ; k^{2} \sin ^{2} \theta\right) d \theta=\int_{0}^{\sin \Phi} \frac{d t}{\sqrt{\left(1-t^{2}\right)\left(1-k^{2} t^{2}\right)}} .
$$

Then

$$
\Phi(z)=z+2 \sum_{n=1}^{\infty} \frac{1}{n} \frac{q^{n}}{1+q^{2 n}} \sin 2 n z .
$$

Proof. Clearly, comparing (1.3) and (1.10),

$$
\operatorname{sn}\left(\theta_{3}^{2} z\right)=\sin \Phi(z) .
$$

Differentiating (1.11) and appealing to the properties (1.1), (1.2) and the Lambert series (1.5), we obtain

$$
\frac{d \Phi}{d z}=\theta_{3}^{2} \frac{c n\left(\theta_{3}^{2} z\right) d n\left(\theta_{3}^{2} z\right)}{\cos \Phi}=\theta_{3}^{2} d n\left(\theta_{3}^{2} z\right)=1+4 \sum_{n=1}^{\infty} \frac{q^{n}}{1+q^{2 n}} \cos 2 n z .
$$

The conclusion follows immediately by integrating (1.12). 
Integrating (1.10) from 0 to $\frac{\pi}{2}$ and using the fact that $\Phi\left(\frac{\pi}{2}\right)=\frac{\pi}{2}$, we have [5] p. 499]

$$
\theta_{3}={ }_{2} F_{1}\left(\frac{1}{2}, \frac{1}{2} ; 1 ; \frac{\theta_{2}^{4}}{\theta_{3}^{4}}\right)
$$

2. Formulation OF THREe FUnCtions BASEd ON ${ }_{2} F_{1}\left(\frac{1}{3}, \frac{2}{3} ; \frac{1}{2} ; z\right)$

Let $0<k_{3}<1$ and let $k_{3}^{\prime}=\sqrt{1-k_{3}^{2}}$. Define

$$
\begin{aligned}
u & =\int_{0}^{\varphi}{ }_{2} F_{1}\left(\frac{1}{3}, \frac{2}{3} ; \frac{1}{2} ; k_{3}^{2} \sin ^{2} \theta\right) d \theta, \\
& =\int_{0}^{\sin \varphi}{ }_{2} F_{1}\left(\frac{1}{3}, \frac{2}{3} ; \frac{1}{2} ; k_{3}^{2} t^{2}\right) \frac{d t}{\sqrt{1-t^{2}}} .
\end{aligned}
$$

Then $\varphi(u)$, with $\varphi(0)=0$, is well defined for $u$ in some neighborhood $U$ of $u=0$. We now define

$$
\begin{aligned}
s n_{3} u & =\sin \varphi \\
c n_{3} u & =\cos \varphi \\
d n_{3} u & =\frac{d \varphi}{d u}=\frac{1}{{ }_{2} F_{1}\left(\frac{1}{3}, \frac{2}{3} ; \frac{1}{2} ; k_{3}^{2} s n_{3}^{2} u\right)}
\end{aligned}
$$

Clearly

$$
s n_{3} 0=0, \quad c n_{3} 0=1, \quad d n_{3} 0=1 .
$$

Moreover, for $u \in U$,

$$
\begin{gathered}
s n_{3}^{2} u+c n_{3}^{2} u=1, \\
k_{3}^{2} s n_{3}^{2} u+\frac{1}{4} d n_{3}^{3} u+\frac{3}{4} d n_{3}^{2} u=1
\end{gathered}
$$

or equivalently,

$$
\begin{gathered}
4 k_{3}^{2} s n_{3}^{2} u=\left(1-d n_{3} u\right)\left(2+d n_{3} u\right)^{2}, \\
s n_{3}^{\prime} u=c n_{3} u d n_{3} u, \\
c n_{3}^{\prime} u=-s n_{3} u d n_{3} u, \\
d n_{3}^{\prime} u=\frac{4}{3} k_{3} c n_{3} u \sqrt{1-d n_{3} u .}
\end{gathered}
$$

Also, $d n_{3} u$ satisfies the following differential equation:

$$
\left(d n_{3}^{\prime} u\right)^{2}=\frac{4}{9}\left(1-d n_{3} u\right)\left(d n_{3}^{3} u+3 d n_{3}^{2} u-4 k_{3}^{\prime 2}\right) .
$$

The properties (2.1), (2.3), and (2.4) follow immediately from the definitions, hence we only need to prove $(2.2),(2.5)$ and $(2.6)$.

We introduce a function $\phi_{3}(u)$ defined by the relation

$$
k_{3} s n_{3} u=\sin \phi_{3}(u), \quad \phi_{3}(0)=0 .
$$

Clearly $\phi_{3}(u)$ is well defined whenever $u$ lies in $U$.

We now prove (2.2). From the identity (1.8),

$$
{ }_{2} F_{1}\left(\frac{1}{3}, \frac{2}{3} ; \frac{1}{2} ; \sin ^{2} z\right)=\frac{\cos \frac{z}{3}}{\cos z} .
$$


Hence

$$
{ }_{2} F_{1}\left(\frac{1}{3}, \frac{2}{3} ; \frac{1}{2} ; \sin ^{2} \phi_{3}\right)=\frac{\cos \frac{\phi_{3}}{3}}{\cos \phi_{3}} .
$$

Thus, from the definition of $d n_{3} u, \cos \frac{\phi_{3}}{3}=\frac{\cos \phi_{3}}{d n_{3} u}$. Appealing to the trigonometric identity

$$
4 \cos ^{3} \frac{\phi_{3}}{3}-3 \cos \frac{\phi_{3}}{3}=\cos \phi_{3}
$$

we obtain

$$
4\left(\frac{\cos \phi_{3}}{d n_{3} u}\right)^{3}-3 \frac{\cos \phi_{3}}{d n_{3} u}=\cos \phi_{3} .
$$

Thus, after simplification,

$$
d n_{3}^{3} u+3 d n_{3}^{2} u=4 \cos ^{2} \phi_{3}=4\left(1-k_{3}^{2} s n_{3}^{2} u\right) .
$$

This proves $(2.2)$.

We now prove (2.5). Differentiating (2.2), we obtain

$$
d n_{3}^{\prime} u=-\frac{8}{3} k_{3}^{2} \frac{s n_{3} u c n_{3} u}{d n_{3} u+2} .
$$

Hence,

$$
\begin{aligned}
\left(d n_{3}^{\prime} u\right)^{2} & =\frac{64}{9} k_{3}^{4} \frac{s n_{3}^{2} u c n_{3}^{2} u}{\left(d n_{3} u+2\right)^{2}} \\
& \left.=\frac{16}{9} k_{3}^{2} \frac{c n_{3}^{2} u\left(d n_{3} u+2\right)^{2}\left(1-d n_{3} u\right)}{\left(d n_{3} u+2\right)^{2}} \quad \text { (by }(2.2)\right) \\
& =\frac{16}{9} k_{3}^{2} c n_{3}^{2} u\left(1-d n_{3} u\right) .
\end{aligned}
$$

This clearly gives $(2.5)$.

The proof of (2.6) goes as follows. From (2.5),

$$
\begin{aligned}
\left(d n_{3}^{\prime} u\right)^{2} & =\frac{16}{9}\left(1-d n_{3} u\right) k_{3}^{2} c n_{3}^{2} u \\
& =\frac{16}{9}\left(1-d n_{3} u\right)\left(k_{3}^{2}-k_{3}^{2} s n_{3}^{2} u\right) \\
& =\frac{4}{9}\left(1-d n_{3} u\right)\left(4 k_{3}^{2}-4+d n_{3}^{3} u+3 d n_{3}^{2} u\right) \\
& =\frac{4}{9}\left(1-d n_{3} u\right)\left(d n_{3}^{3} u+3 d n_{3}^{2} u-4{k_{3}^{\prime}}^{2}\right) .
\end{aligned}
$$

All these identities are valid for $u \in U$. In next section, we will represent $d n_{3} u$ in terms of the Weierstrauss $\wp$ function. This will allow us to replace the above neighborhood $U$ with the entire complex plane.

\section{Representation of $d n_{3}$ By the Weierstrass $\wp$ FUnCtion}

From the fact that $d n_{3} 0=1$ and the differential equation (2.6), we have

$$
u=\frac{3}{2} \int_{1}^{d n_{3} u} \frac{d t}{\sqrt{(1-t)\left(t^{3}+3 t^{2}-4 k_{3}^{\prime 2}\right)}} .
$$


Using the standard formula [5. p. 453] which expresses the above integral in terms of the Weierstrass $\wp$ function, we obtain

$$
d n_{3} u=1-\frac{4}{9} k_{3}^{2}\left(\wp\left(u ; g_{2}, g_{3}\right)+\frac{1}{3}\right)^{-1},
$$

where

and

$$
g_{2}=\frac{4}{27}\left(1+8 k_{3}^{\prime 2}\right)=\frac{4}{27}\left(9-8 k_{3}^{2}\right)
$$

$$
g_{3}=\frac{8}{27^{2}}\left(8{k_{3}^{\prime}}^{4}+20{k_{3}^{\prime}}^{2}-1\right)=\frac{8}{27^{2}}\left(8 k_{3}^{4}-36 k_{3}^{2}+27\right) .
$$

Moreover, straightforward computation yields the discriminant

$$
\Delta\left(g_{2}, g_{3}\right)=g_{2}^{3}-27 g_{3}^{2}=\frac{2^{12}}{3^{9}}\left(1-k_{3}^{2}\right) k_{3}^{6}
$$

and the invariant

$$
J=\frac{g_{2}^{3}-27 g_{3}^{2}}{g_{2}^{3}}=\frac{64\left(1-k_{3}^{2}\right) k_{3}^{6}}{\left(9-8 k_{3}^{2}\right)^{3}} .
$$

Since $\wp(u)$ is meromorphic on the complex plane, we see that, from (3.2), the earlier restriction of $u \in U$ for $d n_{3} u$ can now be removed by the standard procedure of analytic continuation and that $d n_{3} u$ is elliptic follows immediately. In Section 6 , we will obtain very explicit representations of $s n_{3} u, c n_{3} u$ and $d n_{3} u$ in terms of the quotients of the theta function $\theta_{1}(z \mid \tau)$. We also remark that for $0<k_{3}<1, J \neq 0$, $\wp(z)$ does not degenerate into a trigonometric function.

We add that since $\wp$ is of order $2, d n_{3} u$ is also an elliptic function of order 2 . (See [5, p. 432] for the definition of the order of an elliptic function.) Also since $\wp$ is even, $d n_{3}(-u)=d n_{3} u$, hence (3.1) can be written as

$$
u=\frac{3}{2} \int_{d n_{3} u}^{1} \frac{d t}{\sqrt{(1-t)\left(t^{3}+3 t^{2}-4{k_{3}^{\prime}}^{2}\right)}} .
$$

We now discuss the periods of $d n_{3} u$ from the perspective of the theory of conformal mappings. Let

$$
s(w)=\frac{3}{2} \int_{1}^{w} \frac{d t}{\sqrt{(1-t)\left(t^{3}+3 t^{2}-4{k_{3}^{\prime}}^{2}\right)}} .
$$

Thus the multi-valued function $s(w)$ is the inverse function of $d n_{3} u$.

We first note that, using elementary differential calculus, it is easy to see that the denominator has four simple real zeros: $1, x_{1},-x_{2}$ and $-x_{3}$; and $-2<-x_{3}<-x_{2}<$ $0<x_{1}<1$. From the elementary properties of theory of conformal mappings, we see that $s(w)$ maps the lower half plane $\operatorname{Im} w<0$ conformally onto a rectangular region $\mathrm{R}$ with vertices $0,-K,-K-K^{\prime}$ and $-K^{\prime}$, where $K>0$ and $K^{\prime}$ is a purely imaginary number with $\operatorname{Im} K^{\prime}>0$. Moreover, we have $s(1)=0, s\left(x_{1}\right)=-K, s\left(-x_{2}\right)=-K-$ $K^{\prime}$ and $s\left(-x_{3}\right)=-K^{\prime}$. We remark that $s(\infty)$ lies on the imaginary axis between 0 and $-K^{\prime}$. Thus, using the fact that $d n_{3} u$ is an even function, we conclude that $d n_{3} 0=1, d n_{3} K=x_{1}, d n_{3}\left(K+K^{\prime}\right)=-x_{2}$ and $d n_{3} K^{\prime}=-x_{3}$, and there exists a pole of $d n_{3} u$ between the line segment between 0 and $K^{\prime}$. Since $\wp\left(z+K^{\prime}\right)=\wp\left(-z+K^{\prime}\right)$, $d n_{3}\left(u+K^{\prime}\right)=d n_{3}\left(-u+K^{\prime}\right)$, therefore there is also a pole between $K^{\prime}$ and $2 K^{\prime}$. We also point out that $s(-2)$ lies on the line segment between 0 and $-K^{\prime}$. For the same reason, there are exactly two points $u_{1}$ and $u_{2}$ between 0 and $2 K^{\prime}$ such that $d n_{3} u_{1}=d n_{3} u_{2}=-2$. For later use, we state 
Lemma 3.1. $d n_{3} u$ is an elliptic function of order 2. Inside the period parallelogram two of its poles are located on the line segment between 0 and $2 K^{\prime}$ and the solutions of the equation $d n_{3} u+2=0$ are also located between 0 and $2 K^{\prime}$.

The fundamental periods of $d n_{3} u$ are $2 K$ and $2 K^{\prime}$. We will let

$$
\frac{K^{\prime}}{K}=\frac{3 \tau}{2}
$$

We remark that this particular choice of the form for the ratio of $K$ and $K^{\prime}$ makes the subsequent identities and formulas simple and elegant.

With abuse of the earlier notation, we will also use $\wp\left(z ; \omega_{1}, \omega_{2}\right)$ to represent the Weierstrass $\wp$ function of periods $\omega_{1}$ and $\omega_{2}$. If $\omega_{1}=\pi$ and $\omega_{2}=\pi \tau$, for some complex number $\tau$, then we simply write $\wp(z \mid \tau)=\wp(z ; \pi, \pi \tau)$.

Let $K_{3}=K, K_{3}^{\prime}=K^{\prime}$ and $a_{3}=\frac{2 K_{3}}{\pi}$. We show that $d n_{3}\left(a_{3} z\right)$ is an elliptic function of periods $\pi$ and $\frac{3 \pi \tau}{2}$. To see this, we note that [5. p. 439, Example 2]

$$
\wp\left(a z ; a \omega_{1}, a \omega_{2}\right)=\frac{1}{a^{2}} \wp\left(z ; \omega_{1}, \omega_{2}\right) .
$$

Then

$$
\begin{aligned}
d n_{3}\left(a_{3} z\right) & =1-\frac{4}{9} k_{3}^{2}\left(\wp\left(a_{3} z ; 2 K_{3}, 2 K_{3}^{\prime}\right)+\frac{1}{3}\right)^{-1} \\
& =1-\frac{4}{9} k_{3}^{2}\left(\wp\left(\frac{2 K_{3} z}{\pi} ; 2 K_{3}, 3 \tau K_{3}\right)+\frac{1}{3}\right)^{-1} \\
& =1-\frac{4}{9} k_{3}^{2}\left(a_{3}^{-2} \wp\left(z ; \pi, \frac{3 \pi \tau}{2}\right)+\frac{1}{3}\right)^{-1}(\text { from (3.4)) } \\
& =1-\frac{4}{9} a_{3}^{2} k_{3}^{2}\left(\wp\left(z \mid \frac{3 \tau}{2}\right)+\frac{a_{3}^{2}}{3}\right)^{-1} .
\end{aligned}
$$

The corresponding $g_{2}$ and $g_{3}$ for $\wp\left(z \mid \frac{3 \tau}{2}\right)$ are

$$
g_{2}=\frac{2^{6}}{3^{3}} \frac{K_{3}^{4}}{\pi^{4}}\left(1+8{k_{3}^{\prime}}^{2}\right)=\frac{2^{6}}{3^{3}} \frac{K_{3}^{4}}{\pi^{4}}\left(9-8 k_{3}^{2}\right)
$$

and

$$
g_{3}=\frac{2^{9}}{3^{6}} \frac{K_{3}^{6}}{\pi^{6}}\left(8{k_{3}^{\prime}}^{4}+20{k_{3}^{\prime}}^{2}-1\right)=\frac{2^{9}}{3^{6}} \frac{K_{3}^{6}}{\pi^{6}}\left(8 k_{3}^{4}-36 k_{3}^{2}+27\right) .
$$

We recall that the Eisenstein series $E_{4}(\tau)$ and $E_{6}(\tau)$ associated with $\wp(z \mid \tau)$ are

$$
\begin{aligned}
& E_{4}(\tau)=\frac{3}{4} g_{2}=1+240 \sum_{n=1}^{\infty} \frac{n^{3} q^{2 n}}{1-q^{2 n}}, \\
& E_{6}(\tau)=\frac{27}{8} g_{3}=1-504 \sum_{n=1}^{\infty} \frac{n^{5} q^{2 n}}{1-q^{2 n}} .
\end{aligned}
$$

Hence, from (3.6) and (3.7), we have the following expressions of Eisenstein series in terms of $k_{3}$ :

$$
E_{4}\left(\frac{3 \tau}{2}\right)=1+240 \sum_{n=1}^{\infty} \frac{n^{3} q^{3 n}}{1-q^{3 n}}=\frac{16}{9} \frac{K_{3}^{4}}{\pi^{4}}\left(9-8 k_{3}^{2}\right)
$$


and

$$
E_{6}\left(\frac{3 \tau}{2}\right)=1-504 \sum_{n=1}^{\infty} \frac{n^{5} q^{3 n}}{1-q^{3 n}}=\frac{64}{27} \frac{K_{3}^{6}}{\pi^{6}}\left(8 k_{3}^{4}-36 k_{3}^{2}+27\right) .
$$

The most intricate part of this work is to determine the precise locations of the two poles of $d n_{3} u$ in the period parallelogram, and we will devote the entire Section 5 to the proof of

Lemma 3.2. The poles of $d n_{3} u$ in the fundamental period parallelogram are $\frac{2 K_{3}^{\prime}}{3}$ and $\frac{4 K_{3}^{\prime}}{3}$.

Clearly, from (3.5), Lemma 3.2 can be re-stated as follows:

$$
\wp\left(\frac{\pi \tau}{2} \mid \frac{3 \tau}{2}\right)=-\frac{1}{3} a_{3}^{2} .
$$

The proof of Lemma 3.2 will require some detailed facts concerning the properties of the Weierstrass $\wp$ function and in next section, we will list pertinent facts about the Weierstrass $\wp$ function and its connections with the theta functions.

\section{Some identities InVOlving the THETA FUnCTIONS AND THE WEIERSTRASS $\wp$ FUNCTION}

We begin by recalling some familiar properties of the theta functions 5 , p. 465 and p. 469]:

$$
\begin{gathered}
\theta_{1}(z \mid \tau)=2 q^{\frac{1}{4}} \sin z \prod_{n=1}^{\infty}\left(1-q^{2 n}\right)\left(1-q^{2 n} e^{2 i z}\right)\left(1-q^{2 n} e^{-2 i z}\right) \\
\theta_{4}(z \mid \tau)=\prod_{n=1}^{\infty}\left(1-q^{2 n}\right)\left(1-q^{2 n-1} e^{2 i z}\right)\left(1-q^{2 n-1} e^{-2 i z}\right) \\
\theta_{1}(z+\pi \tau \mid \tau)=-q^{-1} e^{-2 i z} \theta_{1}(z \mid \tau) \\
\theta_{1}(z+\pi \mid \tau)=-\theta_{1}(z \mid \tau) .
\end{gathered}
$$

The logarithmic derivatives of $\theta_{1}(z \mid \tau)$ and $\theta_{4}(z \mid \tau)$ are related by

$$
\frac{\theta_{1}^{\prime}}{\theta_{1}}\left(z+\frac{\pi \tau}{2} \mid \tau\right)=\frac{\theta_{4}^{\prime}}{\theta_{4}}(z \mid \tau)-i .
$$

The Lambert series for $\frac{\theta_{4}^{\prime}}{\theta_{4}}(z \mid \tau)$ is [5, p. 489]

$$
\frac{\theta_{4}^{\prime}}{\theta_{4}}(z \mid \tau)=4 \sum_{n=1}^{\infty} \frac{q^{n}}{1-q^{2 n}} \sin 2 n z,
$$

and for $\frac{\theta_{1}^{\prime}}{\theta_{1}}(z \mid \tau)$ is

$$
i \frac{\theta_{1}^{\prime}}{\theta_{1}}(z \mid \tau)=1+2 \sum_{\substack{n=-\infty \\ n \neq 0}}^{\infty} \frac{e^{2 i n z}}{1-q^{2 n}},
$$

where $|q|<\left|e^{i z}\right|<1$. 
The following identities provide connections between the theta functions and the Weierstrass $\wp$ function:

$$
\begin{gathered}
\wp(a \mid \tau)-\wp(z \mid \tau)=\left(\theta_{1}^{\prime}(0 \mid \tau)\right)^{2} \frac{\theta_{1}(z+a \mid \tau) \theta_{1}(z-a \mid \tau)}{\theta_{1}^{2}(z \mid \tau) \theta_{1}^{2}(a \mid \tau)}, \\
\wp^{\prime}(z \mid \tau)=\left(\theta_{1}^{\prime}(0 \mid \tau)\right)^{3} \frac{\theta_{1}(2 z \mid \tau)}{\theta_{1}^{4}(z \mid \tau)}, \\
\wp^{\prime}(z \mid \tau)=-\left(\frac{\theta_{1}^{\prime}}{\theta_{1}}\right)^{\prime \prime}(z \mid \tau), \\
\frac{\theta_{1}^{\prime}}{\theta_{1}}(z+a \mid \tau)-\frac{\theta_{1}^{\prime}}{\theta_{1}}(z-a \mid \tau)-2 \frac{\theta_{1}^{\prime}}{\theta_{1}}(a \mid \tau)=\frac{-\wp^{\prime}(a \mid \tau)}{\wp(z \mid \tau)-\wp(a \mid \tau)}, \\
2 i \frac{\theta_{1}^{\prime}}{\theta_{1}}(a \mid \tau)-\frac{i \wp^{\prime}(a \mid \tau)}{\wp(z \mid \tau)-\wp(a \mid \tau)}=2\left(1+2 \sum_{n=1}^{\infty} \frac{e^{2 i n a}-q^{2 n} e^{-2 i n a}}{1-q^{2 n}} \cos 2 n z\right) .
\end{gathered}
$$

We remark that (4.8) and (4.9) are given, respectively, in [5, p. 451, example 1] and [5, p. 459, Example 24] as the quotients of the Weierstrass Sigma functions, the derivation of (4.10) in the setting of the Weierstrass Sigma function is given in [5. p. 445] and (4.11) is precisely the integral of the identity given in [5, p. 456, example 1].

The proof of (4.12) is as follows.

We note that from (4.3), we have

$$
\frac{\theta_{1}^{\prime}}{\theta_{1}}(z+\pi \tau \mid \tau)=-2 i+\frac{\theta_{1}^{\prime}}{\theta_{1}}(z \mid \tau)
$$

and

$$
\frac{\theta_{1}^{\prime}}{\theta_{1}}(z-a+\pi \tau \mid \tau)=-2 i+\frac{\theta_{1}^{\prime}}{\theta_{1}}(z-a \mid \tau) .
$$

Hence, from (4.13) and (4.7),

$$
\begin{aligned}
i \frac{\theta_{1}^{\prime}}{\theta_{1}}(z+a \mid \tau) & -i \frac{\theta_{1}^{\prime}}{\theta_{1}}(z-a \mid \tau) \\
& =2+i \frac{\theta_{1}^{\prime}}{\theta_{1}}(z+a \mid \tau)-i \frac{\theta_{1}^{\prime}}{\theta_{1}}(z-a+\pi \tau \mid \tau) \\
& =2\left(1+\sum_{\substack{n=-\infty \\
n \neq 0}}^{\infty} \frac{e^{2 i n a} e^{2 i n z}}{1-q^{2 n}}-\frac{e^{-2 i n a} e^{2 i n z} q^{2 n}}{1-q^{2 n}}\right) \\
& =2\left(\begin{array}{c}
\left.1+\sum_{n=-\infty}^{\infty} \frac{e^{2 i n a} e^{2 i n z}}{1-q^{2 n}}-\frac{e^{2 i n a} e^{-2 i n z} q^{-2 n}}{1-q^{-2 n}} \frac{q^{2 n}}{q^{2 n}}\right) \\
=
\end{array}\right) \\
& =2\left(\begin{array}{c}
1+2 \sum_{n=-\infty}^{\infty} \frac{e^{2 i n a}}{1-q^{2 n}} \cos 2 n z \\
n \neq 0
\end{array}\right)
\end{aligned}
$$


Equation (4.12) now follows immediately from (4.11) and (4.14).

For subsequent use, we now list the values of $\wp^{\prime}\left(z \mid \frac{3 \tau}{2}\right)$ at various points. From (4.1) and (4.9), it is easy to deduce that

$$
\wp^{\prime}\left(\frac{\pi \tau}{2} \mid \frac{3 \tau}{2}\right)=\frac{-8 i}{27} c_{3}^{3}
$$

and

$$
\wp^{\prime}\left(\frac{\pi}{2} \mid \frac{\tau}{2}\right)=-\frac{8}{3^{\frac{3}{2}}} b_{3}^{3}
$$

where

$$
c_{3}=3 q^{\frac{1}{3}} \prod_{n=1}^{\infty} \frac{\left(1-q^{3 n}\right)^{3}}{\left(1-q^{n}\right)} \quad \text { and } \quad b_{3}=\prod_{n=1}^{\infty} \frac{\left(1-q^{n}\right)^{3}}{\left(1-q^{3 n}\right)} .
$$

It is interesting to comment that the Lambert series for $c_{3}^{3}$ can be derived quite easily from our current setting. To see this, we note, from (4.5) and (4.10), that

$$
\wp^{\prime}\left(z+\frac{\pi \tau}{2} \mid \tau\right)=-\left(\frac{\theta_{1}^{\prime}}{\theta_{1}}\right)^{\prime \prime}\left(z+\frac{\pi \tau}{2} \mid \tau\right)=-\left(\frac{\theta_{4}^{\prime}}{\theta_{4}}\right)^{\prime \prime}(z \mid \tau) .
$$

Thus, from (4.17) and the second derivative of the Lambert series (4.6),

$$
\wp^{\prime}\left(\frac{\pi \tau}{2} \mid \frac{3 \tau}{2}\right)=\left(\frac{\theta_{4}^{\prime}}{\theta_{4}}\right)^{\prime \prime}\left(\frac{\pi \tau}{4} \mid \frac{3 \tau}{2}\right)=-8 i \sum_{n=1}^{\infty} \frac{n^{2} q^{n}}{1+q^{n}+q^{2 n}} .
$$

Hence, we have the Lambert series for $c_{3}^{3}$ :

$$
c_{3}^{3}=27 \sum_{n=1}^{\infty} \frac{n^{2} q^{n}}{1+q^{n}+q^{2 n}} .
$$

For later use, we also need the following fact which follows readily from (4.7):

$$
3 i \frac{\theta_{1}^{\prime}}{\theta_{1}}\left(\frac{\pi \tau}{2} \mid \frac{3 \tau}{2}\right)-2=1+6 \sum_{n=1}^{\infty} \frac{q^{n}}{1+q^{n}+q^{2 n}} .
$$

\section{Proof of Lemma 3.2}

The proof will be done in five steps.

Step 1. Let $\Phi(z)=\varphi\left(a_{3} z\right)$. We first express (3.5) in terms $\Phi$ and $\theta_{1}$, and from which we derive

$$
\Phi(z)=\left(a_{3}-2 i \alpha \frac{\theta_{1}^{\prime}}{\theta_{1}}\left(a \mid \frac{3 \tau}{2}\right)+2 \alpha\right) z+2 \alpha \sum_{n=1}^{\infty} \frac{1}{n} \frac{e^{2 i n a}-q^{3 n} e^{-2 i n a}}{1-q^{3 n}} \sin 2 n z,
$$

where $\alpha=-\frac{4 i}{9} \frac{a_{3}^{3} k_{3}^{2}}{\wp^{\prime}\left(a \mid \frac{3 \tau}{2}\right)}$ and $a$ is a point in the period parallelogram of $\wp\left(z \mid \frac{3 \tau}{2}\right)$ such that $\wp\left(a \mid \frac{3 \tau}{2}\right)=-\frac{a_{3}^{2}}{3}$. Hence $a$ is a pole of $d n_{3}\left(a_{3} z\right)$. From Lemma 3.1, we know that one of the poles of $d n_{3} u$ lies on the line segment between 0 and $K^{\prime}$; we can therefore assume that $a$ lies on the line segment between 0 and $\frac{3 \pi \tau}{4}$.

Recall that

$$
d n_{3} u=\frac{d \varphi}{d u}
$$

then

$$
\Phi^{\prime}(z)=a_{3} d n_{3} u
$$


Hence, from (3.5),

$$
\Phi^{\prime}(z)=a_{3}-\frac{4}{9} a_{3}^{3} k_{3}^{2}\left(\wp\left(z \mid \frac{3 \tau}{2}\right)+\frac{a_{3}^{2}}{3}\right)^{-1}=a_{3}+i \alpha \frac{-\wp^{\prime}\left(a \mid \frac{3 \tau}{2}\right)}{\wp\left(z \mid \frac{3 \tau}{2}\right)-\wp\left(a \mid \frac{3 \tau}{2}\right)} .
$$

Then from (4.12)

$$
\begin{aligned}
\Phi^{\prime}(z) & =a_{3}-2 i \alpha \frac{\theta_{1}^{\prime}}{\theta_{1}}\left(a \mid \frac{3 \tau}{2}\right)+i \alpha\left(\frac{\theta_{1}^{\prime}}{\theta_{1}}\left(z+a \mid \frac{3 \tau}{2}\right)-\frac{\theta_{1}^{\prime}}{\theta_{1}}\left(z-a \mid \frac{3 \tau}{2}\right)\right) \\
& =a_{3}-2 i \alpha \frac{\theta_{1}^{\prime}}{\theta_{1}}\left(a \mid \frac{3 \tau}{2}\right)+2 \alpha+4 \alpha \sum_{n=1}^{\infty} \frac{e^{2 i n a}-q^{3 n} e^{-2 i n a}}{1-q^{3 n}} \cos 2 n z
\end{aligned}
$$

Integrating (5.3), we obtain the desired conclusion.

Step 2. We now make a further simplification of (5.1):

$$
\Phi(z)=z+2 \alpha \sum_{n=1}^{\infty} \frac{1}{n} \frac{e^{2 i n a}-q^{3 n} e^{-2 i n a}}{1-q^{3 n}} \sin 2 n z .
$$

We write $(2.2)$ in terms of $\Phi$ :

$$
4 k_{3}^{2} \sin ^{2} \Phi(z)=\left(1-d n_{3} a_{3} z\right)\left(2+d n_{3} a_{3} z\right)^{2} .
$$

Since $\pi$ is a period of $d n_{3} a_{3} z, d n_{3} a_{3} \pi=d n_{3} 0=1$. Hence $\sin \Phi(\pi)=0$ and, from (5.5), this implies that $\Phi(\pi)=N \pi$ for some integer $N$. We will prove that $N=1$.

Suppose $|N|>1$.

We observe that, from (5.2), $\Phi^{\prime}(x)$ is real valued if $x$ is real, thus $\Phi(x)$ is real on the real axis. Since $\Phi(0)=0$ and $\Phi(\pi)=N \pi$, we conclude from the intermediate value theorem that there exists an $x^{*}$ with $0<x^{*}<\pi$ (or a $u^{*}$ with $0<u^{*}<2 K$ ) such that $\Phi\left(x^{*}\right)=\pi$ or $-\pi$. This in turn implies that $\sin \Phi\left(x^{*}\right)=0$ and we conclude from (5.5) that either $d n_{3} u^{*}=1$ or $d n_{3} u^{*}=-2$. Since $d n_{3} u$ is an elliptic function of order 2 , the equation $d n_{3} u-1=0$ has exactly two solutions in the period parallelogram and from (5.2) we see that it already has double zeros at $u=0$, hence $d n_{3} u^{*}=1$ will yield a third solution and this leads to a contradiction.

Now consider that case $d n_{3} u^{*}=-2$. From Lemma 3.1, $d n_{3} u+2=0$ has two solutions on the line segment between 0 and $2 K^{\prime}$, hence a third solution $u^{*}$ between 0 and $2 K$ again leads to the same contradiction.

Therefore either $N=1$ or $N=-1$.

Suppose $N=-1$. Then $\Phi(\pi)=-\pi$. Since $\Phi(0)=0$ and $\Phi^{\prime}(0)=a_{3}>0$, we conclude that there exists an $x^{*}$ between 0 and $\pi$ such that $\Phi\left(x^{*}\right)=0$. This again leads to the same contradiction.

Thus $N=1$.

Since $\Phi(\pi)=\pi$, we conclude from (5.1) that

$$
a_{3}-2 i \alpha \frac{\theta_{1}^{\prime}}{\theta_{1}}\left(a \mid \frac{3 \tau}{2}\right)+2 \alpha=1 .
$$

Substituting (5.6) into (5.3), then the desired conclusion (5.4) follows from integration of (5.3).

Step 3. $\alpha=\frac{3}{2}$.

Using (5.6), we can re-write the first equation of (5.3) as

$$
\Phi^{\prime}(z)=1-2 \alpha+i \alpha\left(\frac{\theta_{1}^{\prime}}{\theta_{1}}\left(z+a \mid \frac{3 \tau}{2}\right)-\frac{\theta_{1}^{\prime}}{\theta_{1}}\left(z-a \mid \frac{3 \tau}{2}\right)\right) .
$$


Integrating (5.7), we obtain, after exponentiation,

$$
e^{-i \Phi(z)}=e^{-i(1-2 \alpha) z}\left(-\frac{\theta_{1}\left(z+a \mid \frac{3 \tau}{2}\right)}{\theta_{1}\left(z-a \mid \frac{3 \tau}{2}\right)}\right)^{\alpha} .
$$

We now prove $\alpha=\frac{3}{2}$. To this end, we re-write (2.2) as

$$
4 K_{3}^{2} \sin ^{2} \Phi=4-\left(\frac{1}{a_{3}} \Phi^{\prime}\right)^{3}-3\left(\frac{1}{a_{3}} \Phi^{\prime}\right)^{2} .
$$

Since the right-hand side of (5.9) is elliptic, we conclude that its left-hand side

$$
4 \sin ^{2} \Phi=2 \cos 2 \Phi-2=e^{2 i \Phi}+e^{-2 i \Phi}-2
$$

is elliptic as well. Since $\Phi^{\prime}$ has poles of order 1, this implies, from (5.9), that $\sin ^{2} \Phi$ has poles of order 3. From (5.8) and (5.10), we conclude that $\alpha=\frac{3}{2}$.

Step 4. $e^{ \pm 2 i \Phi}$ is elliptic and of periods $\pi$ and $\frac{3 \pi \tau}{2}$.

To see this, we differentiate (5.9). Then

$$
4 k_{3} \sin 2 \Phi=-\frac{3}{a_{3}^{3}} \Phi^{\prime} \Phi^{\prime \prime}-\frac{6}{a_{3}^{2}} \Phi^{\prime \prime} .
$$

This shows that $\sin 2 \Phi$ is elliptic. From (5.10), $\cos 2 \Phi$ is elliptic, therefore $e^{ \pm 2 i \Phi}=$ $\cos 2 \Phi \pm i \sin 2 \Phi$ is elliptic.

Step 5 . We now prove $a=\frac{\pi \tau}{2}$.

Let

$$
f(z)=-e^{-2 i \Phi} .
$$

Then, from (5.8),

$$
f(z)=e^{4 i z}\left(\frac{\theta_{1}\left(z+a \mid \frac{3 \tau}{2}\right)}{\theta_{1}\left(z-a \mid \frac{3 \tau}{2}\right)}\right)^{3} .
$$

Using the properties (4.3) and (4.4) of $\theta_{1}(z \mid \tau)$, we have

$$
f\left(z+\frac{3 \pi \tau}{2}\right)=e^{4 i\left(z+\frac{3 \pi \tau}{2}\right)}\left(\frac{\theta_{1}\left(z+a+\frac{3 \pi \tau}{2} \mid \frac{3 \tau}{2}\right)}{\theta_{1}\left(z-a+\frac{3 \pi \tau}{2} \mid \frac{3 \tau}{2}\right)}\right)^{3}=q^{6} e^{-12 i a} f(z) .
$$

Since $f$ has period $\frac{3 \pi \tau}{2}$, therefore $a=\frac{\pi \tau}{2}+\frac{n \pi}{6}$ for some integer $n$. We have earlier proved that the pole of $d n_{3} u$ is purely imaginary, hence we conclude that $n=0$ and this completes the proof of Step 5.

Substituting the values of $\alpha$ and $a$ into (5.4), we conclude that

$$
\Phi(z)=z+3 \sum_{n=1}^{\infty} \frac{1}{n} \frac{q^{n}}{1+q^{n}+q^{2 n}} \sin 2 n z .
$$

6. Representations of $s n_{3} u, c n_{3}$ AND $d n_{3} u$ AS QUOTIENTS of $\theta_{1}$

Since $s n_{3}\left(a_{3} z\right)=\sin \Phi(z)$ and $c n_{3}\left(a_{3} z\right)=\cos \Phi(z)$, we conclude from Step 5 that

$$
s n_{3}\left(a_{3} z\right)=\frac{1}{2}\left(e^{2 i z}\left(\frac{\theta_{1}\left(z+\frac{\pi \tau}{2} \mid \frac{3 \tau}{2}\right)}{\theta_{1}\left(z-\frac{\pi \tau}{2} \mid \frac{3 \tau}{2}\right)}\right)^{3 / 2}+e^{-2 i z}\left(\frac{\theta_{1}\left(z-\frac{\pi \tau}{2} \mid \frac{3 \tau}{2}\right)}{\theta_{1}\left(z+\frac{\pi \tau}{2} \mid \frac{3 \tau}{2}\right)}\right)^{3 / 2}\right)
$$

and

$$
c n_{3}\left(a_{3} z\right)=\frac{1}{2 i}\left(e^{2 i z}\left(\frac{\theta_{1}\left(z+\frac{\pi \tau}{2} \mid \frac{3 \tau}{2}\right)}{\theta_{1}\left(z-\frac{\pi \tau}{2} \mid \frac{3 \tau}{2}\right)}\right)^{3 / 2}-e^{-2 i z}\left(\frac{\theta_{1}\left(z-\frac{\pi \tau}{2} \mid \frac{3 \tau}{2}\right)}{\theta_{1}\left(z+\frac{\pi \tau}{2} \mid \frac{3 \tau}{2}\right)}\right)^{3 / 2}\right) .
$$


We remark that $s n_{3} u$ and $c n_{3} u$ are not elliptic; however, $s n_{3}^{2} u$ and $c n_{3}^{2} u$ are both elliptic.

We now express $d n_{3}\left(a_{3} z\right)$ in terms of $\theta_{1}$.

First we recall the identity (4.8):

$$
\wp(a \mid \tau)-\wp(z \mid \tau)=\left(\theta_{1}^{\prime}(0 \mid \tau)\right)^{2} \frac{\theta_{1}(z+a \mid \tau) \theta_{1}(z-a \mid \tau)}{\theta_{1}^{2}(z \mid \tau) \theta_{1}^{2}(a \mid \tau)} .
$$

From (5.2) and the above identity with $a=\frac{\pi \tau}{2}$, we obtain

$$
d n_{3}\left(a_{3} z\right)=1+\frac{3 i}{2} \frac{1}{a_{3}} \frac{\theta_{1}\left(\pi \tau \mid \frac{3 \tau}{2}\right) \theta_{1}^{\prime}\left(0 \mid \frac{3 \tau}{2}\right)}{\theta_{1}^{2}\left(\pi \tau \mid \frac{3 \tau}{2}\right)} \frac{\theta_{1}^{2}\left(z \mid \frac{3 \tau}{2}\right)}{\theta_{1}\left(z+\frac{\pi \tau}{2} \mid \frac{3 \tau}{2}\right) \theta_{1}\left(z-\frac{\pi \tau}{2} \mid \frac{3 \tau}{2}\right)} .
$$

From (4.1), we obtain

$$
\begin{gathered}
\theta_{1}\left(\pi \tau \mid \frac{3 \tau}{2}\right)=q^{-1 / 2} \theta_{1}\left(\frac{\pi \tau}{2} \mid \frac{3 \tau}{2}\right), \\
\theta_{1}\left(\frac{\pi \tau}{2} \mid \frac{3 \tau}{2}\right)=i q^{-1 / 8} \prod_{n=1}^{\infty}\left(1-q^{n}\right),
\end{gathered}
$$

and

$$
\theta_{1}^{\prime}\left(0 \mid \frac{3 \tau}{2}\right)=2 q^{3 / 8} \prod_{n=1}^{\infty}\left(1-q^{3 n}\right)^{3} .
$$

Substituting these quantities into (6.1), we have

$$
d n_{3}\left(a_{3} z\right)=1+q^{-1 / 3} \frac{c_{3}}{a_{3}} \frac{\theta_{1}^{2}\left(z \mid \frac{3 \tau}{2}\right)}{\theta_{1}\left(z+\frac{\pi \tau}{2} \mid \frac{3 \tau}{2}\right) \theta_{1}\left(z-\frac{\pi \tau}{2} \mid \frac{3 \tau}{2}\right)},
$$

where $c_{3}$ is defined as in (4.15).

\section{Counterparts of the Jacobian ElLiptic FunCtions}

Differentiating (5.12), we obtain the Lambert series for $a_{3} d n_{3} a_{3} z$ :

$$
a_{3} d n_{3} a_{3} z=1+6 \sum_{n=1}^{\infty} \frac{q^{n}}{1+q^{n}+q^{2 n}} \cos 2 n z .
$$

We now express $a_{3}$ as a Lambert series: With $\alpha=\frac{3}{2}$ and $a=\frac{\pi \tau}{2}$, we obtain from (5.6) and (4.19),

$$
a_{3}=-2+3 i \frac{\theta_{1}^{\prime}}{\theta_{1}}\left(\frac{\pi \tau}{2} \mid \frac{3 \tau}{2}\right)=1+6 \sum_{n=1}^{\infty} \frac{q^{n}}{1+q^{n}+q^{2 n}} ;
$$

and from (4.15) and the definition of $\alpha$ in (5.1), we derive

$$
k_{3}^{2}=\frac{c_{3}^{3}}{a_{3}^{3}} .
$$

Since $\sin \Phi(x)=s n_{3} \frac{2 K_{3} x}{\pi}$ and $\Phi\left(\frac{\pi}{2}\right)=\frac{\pi}{2}$,

$$
s n_{3} K_{3}=1 \text {. }
$$

Then, from (2.2) with $u=K_{3}$, we have

$$
1-k_{3}^{2}=\frac{1}{4} d n_{3}^{3} K_{3}+\frac{3}{4} d n_{3}^{2} K_{3} .
$$


Solving the above cubic equation, we obtain

$$
d n_{3} K_{3}=\left(1-2 k_{3}^{2}+2 i k_{3} k_{3}^{\prime}\right)^{\frac{1}{3}}+\left(1-2 k_{3}^{2}+2 i k_{3} k_{3}^{\prime}\right)^{-\frac{1}{3}}-1 .
$$

We now introduce the abbreviated notation: $a=a_{3}, b=b_{3}$ and $c=c_{3}$, and show that the identity (7.3) is precisely the Borweins' Cubic identity [1]:

$$
a^{3}=b^{3}+c^{3} .
$$

To see this, we recall the fact that $d n_{3} K_{3}=\frac{1}{a_{3}} \Phi^{\prime}\left(\frac{\pi}{2}\right)$. Then (7.3) can be rewritten as

$$
1-k_{3}^{2}=\frac{1}{4}\left(\frac{1}{a_{3}} \Phi^{\prime}\left(\frac{\pi}{2}\right)\right)^{3}+\frac{3}{4}\left(\frac{1}{a_{3}} \Phi^{\prime}\left(\frac{\pi}{2}\right)\right)^{2} .
$$

We also need two identities involving $\Phi^{\prime}\left(\frac{\pi}{2}\right)$ :

$$
\begin{gathered}
\Phi^{\prime}\left(\frac{\pi}{2}\right)=\prod_{n=1}^{\infty} \frac{\left(1-q^{n}\right)^{3}}{\left(1-q^{3 n}\right)} \frac{\left(1+q^{3 n}\right)}{\left(1+q^{n}\right)^{3}}, \\
\Phi^{\prime}\left(\frac{\pi}{2}\right)+3 a=4 \prod_{n=1}^{\infty} \frac{\left(1-q^{n}\right)^{3}}{\left(1-q^{3 n}\right)} \frac{\left(1+q^{n}\right)^{6}}{\left(1+q^{3 n}\right)^{2}} .
\end{gathered}
$$

To prove (7.5), we note from (5.12),

$$
\Phi^{\prime}\left(\frac{\pi}{2}\right)=1+6 \sum_{n=1}^{\infty} \frac{(-1)^{n} q^{n}}{1+q^{n}+q^{2 n}} .
$$

Then

$$
\begin{aligned}
\Phi^{\prime}\left(\frac{\pi}{2}\right) & =1+6 \sum_{n=1}^{\infty} \frac{(-1)^{n} q^{n}}{1+q^{n}+q^{2 n}} \\
& =1+6 \sum_{n=1}^{\infty} \frac{(-1)^{n} q^{n}}{1+q^{n}+q^{2 n}} \frac{1-q^{n}}{1-q^{n}} \\
& =1+6 \sum_{n=1}^{\infty} \frac{(-1)^{n}\left(q^{n}-q^{2 n}\right)}{1-q^{3 n}} \\
& =1+6 \sum_{n=1}^{\infty}(-1)^{n}\left(q^{n}-q^{2 n}\right) \sum_{k=0}^{\infty} q^{3 n k} \\
& =1+6 \sum_{k=0}^{\infty} \sum_{n=1}^{\infty}(-1)^{n}\left(q^{(3 k+1) n}-q^{(3 k+2) n}\right) \\
& =1-6 \sum_{k=0}^{\infty} \frac{q^{3 k+1}}{1+q^{3 k+1}}-\frac{q^{3 k+2}}{1+q^{3 k+2}} \\
& =\prod_{n=1}^{\infty} \frac{\left(1-q^{n}\right)^{3}}{\left(1-q^{3 n}\right)} \frac{\left(1+q^{3 n}\right)}{\left(1+q^{n}\right)^{3}} \quad[4 \text { p. 1105, eq. (3.6)]. }
\end{aligned}
$$


Equation (7.6) is derived as follows:

$$
\begin{aligned}
& \Phi^{\prime}\left(\frac{\pi}{2}\right)+3 a=4+6 \sum_{n=1}^{\infty} \frac{(-1)^{n} q^{n}}{1+q^{n}+q^{2 n}}+18 \sum_{n=1}^{\infty} \frac{q^{n}}{1+q^{n}+q^{2 n}} \\
& =4+24 \sum_{n, \text { even }}^{\infty} \frac{q^{n}}{1+q^{n}+q^{2 n}}+12 \sum_{n, \text { odd }}^{\infty} \frac{q^{n}}{1+q^{n}+q^{2 n}} \\
& =4+12 \sum_{n, \text { even }}^{\infty} \frac{q^{n}}{1+q^{n}+q^{2 n}}+12 \sum_{n=1}^{\infty} \frac{q^{n}}{1+q^{n}+q^{2 n}} \\
& =4\left(1+3 \sum_{n=1}^{\infty} \frac{q^{2 n}\left(1-q^{2 n}\right)}{1-q^{6 n}}+3 \sum_{n=1}^{\infty} \frac{q^{n}\left(1-q^{n}\right)}{1-q^{3 n}}\right) \\
& =4\left(1+3 \sum_{n=0}^{\infty} \frac{q^{6 n+2}}{1-q^{6 n+2}}-\frac{q^{6 n+4}}{1-q^{6 n+4}}+3 \sum_{n=0}^{\infty} \frac{q^{3 n+1}}{1-q^{3 n+1}}-\frac{q^{3 n+2}}{1-q^{3 n+2}}\right) \\
& =4\left(1+3 \sum_{n=0}^{\infty} \frac{q^{6 n+2}}{1-q^{6 n+2}}-\frac{q^{6 n+4}}{1-q^{6 n+4}}+3 \sum_{n=0}^{\infty} \frac{q^{6 n+1}}{1-q^{6 n+1}}+\frac{q^{6 n+4}}{1-q^{6 n+4}}\right. \\
& \left.-3 \sum_{n=0}^{\infty} \frac{q^{6 n+2}}{1-q^{6 n+2}}+\frac{q^{6 n+5}}{1-q^{6 n+5}}\right) \\
& =4\left(1+3 \sum_{n=}^{\infty} \frac{q^{6 n+1}}{1-q^{6 n+1}}-\frac{q^{6 n+5}}{1-q^{6 n+5}}\right) \\
& =4 \prod_{n=1}^{\infty} \frac{\left(1-q^{n}\right)^{3}}{\left(1-q^{3 n}\right)} \frac{\left(1+q^{n}\right)^{6}}{\left(1+q^{3 n}\right)^{2}} \quad \text { [4. p. 1104, eq. (3.1)]. }
\end{aligned}
$$

Multiplying (7.5) and (7.6), we obtain

$$
\frac{1}{4}\left(\Phi^{\prime}\left(\frac{\pi}{2}\right)\right)^{2}\left(\Phi^{\prime}\left(\frac{\pi}{2}\right)+3 a\right)=\left(\prod_{n=1}^{\infty} \frac{\left(1-q^{n}\right)^{3}}{\left(1-q^{3 n}\right)}\right)^{3}=b_{3}^{3} .
$$

The Borweins' Cubic identity now follows by multiplying (7.4) with $a^{3}$ :

$$
a^{3}-c^{3}=\frac{1}{4}\left(\Phi^{\prime}\left(\frac{\pi}{2}\right)\right)^{2}\left(\Phi^{\prime}\left(\frac{\pi}{2}\right)+3 a\right)=b^{3} .
$$

We end the section with

Theorem 7.1. Let $0<k<1$.

(1) Then there exists a purely imaginary number $\tau$ with $\operatorname{Im} \tau>0$ such that $k=\sqrt{\frac{c^{3}}{a^{3}}}$.

(2) (Ramanujan) If we define

$$
a z=\int_{0}^{\Phi}{ }_{2} F_{1}\left(\frac{1}{3}, \frac{2}{3} ; \frac{1}{2} ; k^{2} \sin ^{2} \theta\right) d \theta,
$$

then

$$
\Phi(z)=1+3 \sum_{n=1}^{\infty} \frac{1}{n} \frac{q^{n}}{1+q^{n}+q^{2 n}} \sin 2 n z .
$$


As a corollary, we have

$$
a={ }_{2} F_{1}\left(\frac{1}{3}, \frac{2}{3} ; 1 ; \frac{c^{3}}{a^{3}}\right) \text {. }
$$

We remark that the major part of the work is devoted to the proof of statement (1); however, if we know a priori that $k^{2}=\frac{c^{3}}{a^{3}}$ for some $\tau$, then it is relatively simple to establish the statement (2). (See [3] for details.)

\section{ACKNowledgement}

I would like to take this opportunity to express my deep gratitude to the referee for contributing many substantial comments and valuable suggestions on the original manuscript to make the work more coherent and readable.

\section{REFERENCES}

1. J. M. Borwein and P. B. Borwein, A cubic counterpart of Jacobi's identity and AGM, Trans. Amer. Math. Soc. 323(1991), 691-701. MF 91e:33012

2. A. Erdelyi (Editor)," Higher Transcendental Functions", Vol. 1, McGraw-Hill, New York, 1953. MR 15:419i

3. Li-Chien Shen, On an identity of Ramanujan based on the hypergeometric series ${ }_{2} F_{1}(1 / 3,2 / 3 ; 1 / 2 ; x)$, J. Number Theory 69(1998), 125-134. MF99d:11042

4. Li-Chien Shen, On the modular equations of degree 3, Proc. Amer. Math. Soc. 122(1994), 1101-1114. MR95b:11044

5. E. T. Whittaker and G. N. Watson, A Course of Modern Analysis, 4th ed., Cambridge University Press, Cambridge, 1966.

Department of Mathematics, University of Florida, Gainesville, Florida 32611-2082

E-mail address: shen@math.ufl.edu 